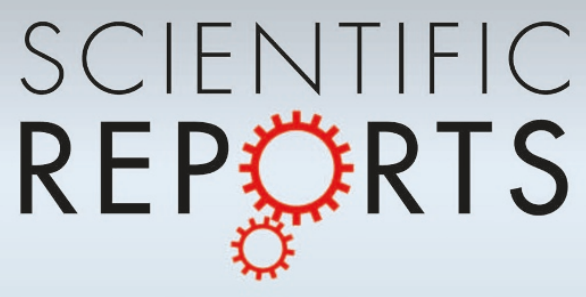

OPEN

SUBJECT AREAS:

COEVOLUTION

SOCIAL EVOLUTION

PHASE TRANSITIONS AND CRITICAL PHENOMENA

Received

22 January 2014

Accepted

16 July 2014

Published

5 August 2014

Correspondence and requests for materials should be addressed to Y.X.L. (yxli@zufe.edu. $\mathrm{cn})$

\section{The evolution of reputation-based} partner-switching behaviors with a cost

\author{
Yixiao Li
}

School of Information, Zhejiang University of Finance and Economics, Hangzhou, Zhejiang 310018, China.

Humans constantly adjust their social relationships and choose new partners of good reputations, thereby promoting the evolution of cooperation. Individuals have to pay a cost to build a reputation, obtain others' information and then make partnership adjustments, yet the conditions under which such costly behaviors are able to evolve remain to be explored. In this model, I assume that individuals have to pay a cost to adjust their partnerships. Furthermore, whether an individual can adjust his partnership based on reputation is determined by his strategic preference, which is updated via coevolution. Using the metaphor of a public goods game where the collective benefit is shared among all members of a group, the coupling dynamics of cooperation and partnership adjustment were numerically simulated. Partner-switching behavior cannot evolve in a public goods game with a low amplification factor. However, such an effect can be exempted by raising the productivity of public goods or the frequency of partnership adjustment. Moreover, costly partner-switching behavior is remarkably promoted by the condition that the mechanism of reputation evaluation considers its prosociality. A mechanism of reputation evaluation that praises both cooperative and partner-switching behaviors allows them to coevolve.

T he prevalence of cooperative or altruistic behaviors among individuals underpins human societies, giving rise to a marvelous complexity of societal and economic organizations ${ }^{1-5}$. Human individuals who are narrowly self-interested to maximize their personal welfare are termed as homo economicus. Although selfishness is a rational choice, a wide range of natural and experimental settings have demonstrated the ubiquity of irrational individuals who are willing to improve the welfare of others, the so-called homo reciprocans ${ }^{6}$. This phenomenon attracts extensive attention from evolutionary biologists, psychologists, economists, etc. In a simple game-theoretical model that characterizes the mutual interplay between individuals, two strategic options are available: defection (D) and cooperation (C). When a population of individuals playing such a game is exposed to natural selection or individuals autonomously imitate fitter ones, cooperation becomes an evolutionarily disadvantaged strategy. Therefore, additional mechanisms are required to sustain the evolution of cooperation. Various mechanisms with the potential to meet this function can be grouped into several categories ${ }^{5}$.

Reputation mechanisms play a crucial role in human cooperation. Increased cognitive capacity allowed recently evolved primates to track reputations in a way that other species could not. For example, only chimpanzees and humans exhibit the capacity to retain information regarding third-party interactions ${ }^{7}$. Interestingly, concern for self-reputation is observed in human children rather than chimpanzees ${ }^{8}$. Humans are often indirectly reciprocal, i.e., I help you and someone else helps me, which requires a building of reputation' ${ }^{9}$ Even among repeated encounters, reputation is vital to establish the cooperative order. An individual's reputation reflects his willingness to cooperate with others. Notably, some second-order altruistic behaviors can create the reputation effect as well, i.e., altruistic punishment ${ }^{10}$. Although an altruistic individual suffers the cost of helping others, he will most likely be recognized and rewarded by other counterparts in the future. In the long run, cooperative behaviors as well as a system of reputation evaluation and tracking can be fostered. Gossip or rumor may contribute to achieving a similar purpose ${ }^{11}$.

In indirect reciprocity models, the central issue is how to evaluate and quantify an individual's behaviors. By considering different levels of information, including the donors' behaviors, the receivers' reputations and the donors' reputations, reputation can be evaluated delicately ${ }^{9,12-14}$. Individuals can take various strategies against others according to their reputations. The evolution of indirect reciprocity depends on praising altruistic behaviors and suppressing selfish behaviors. Rockenbach and Milinski found in a human experiment that the frequency of costly punishment acts was reduced to a low level in the presence of indirect reciprocity ${ }^{15}$. A theoretical study showed that there is only a small parameter region where costly punishment is more efficient than strategies of indirect reciprocity ${ }^{16}$. An empirical study conducted in a Peruvian highland community indicates that persons who contributed more to the collective action had better reputations for various qualities. 
Larger support networks and healthier states distinguished households of greater reputations ${ }^{17}$. However, the cost of reputation building and the observability of reputation information are the factors that condition the success of indirect reciprocity ${ }^{18,19}$. A recent experiment produced the interesting result that money can serve as a symbol of trust among strangers, i.e., an analog of reputation ${ }^{20}$. In their experiment, subjects gave worthless tokens to reward others' help and demanded a token in exchange for help. Trust among strangers could be developed as the social groups grew larger and tokens endogenously became money.

With the aid of reputation, cooperation can be enhanced in network reciprocity models. When interaction patterns among individuals are structured as a network, the equilibrium frequency of cooperation in the whole population exhibits a second-order phase transition as the benefit-to-cost ratio increases ${ }^{21,22}$. Such a mechanism is referred to as network reciprocity, and such games played by networked individuals are referred to as spatial games or networked games. Researchers have found that the complement of reputation mechanisms favors cooperation in network reciprocity models. Brandt et al. studied the effect of reputation in spatial public goods games $(\mathrm{PGGs})^{23}$. In the presence of reputation, highly cooperative and fair outcomes are achieved. Wang et al. proposed a partner selection rule on the basis of evaluating both reputations of a pair in a spatial $\mathrm{PGG}^{24}$. If two adjacent players' reputations are within the tolerance range, the physical connection can be transformed into an interaction relationship. In this setting, a moderate tolerance range of reputation results in the best environments for cooperation.

In the real world, human social networks are relatively static at the macroscopic scale, whereas individuals frequently adjust their social relationships at the local level. Therefore, partnership dynamics must be incorporated into spatial game models. Zimmermann et al. proposed a model where a spatial game is played by agents and the network of interaction links evolves to adapt to the outcome of the game $^{25}$. A cooperator can dismiss an interaction with a defector and switch to another randomly chosen agent. This activity triggers the emergence of highly connected nodes and a highly cooperative stationary state. More recently, the coevolution of cooperation and partnership networks has led to a considerable amount of experimental and theoretical investigation ${ }^{26-35}$.

Fu et al. introduced the mechanism of reputation-based partner choice into the spatial prisoner's dilemma game ${ }^{36}$. Individuals can either change their strategies by imitating their partners or adjust their partnerships based on local information regarding reputations. Such a mechanism of partner switching based on reputation brings about a significant promotion of cooperation with the evolution of a heterogeneous partnership network. When individuals play PGGs, the stationary density of cooperators depends on the group size, and reputation-based partner selection can improve cooperation remark$\mathrm{ably}^{37}$. In an experimental setting, Sylwester and Roberts showed that cooperators benefit from these reputation-based partner-switching behaviors ${ }^{38}$. Even if agents are restricted to adjusting within their geographical neighborhood, the reputation-based partner choice promotes cooperation efficiently ${ }^{39}$. It is worth pointing out that unilateral reputation-based partner switching in a non-excludable PGG cannot lead to the promotion of cooperation ${ }^{40}$.

The puzzle of rampant cooperation in humans lies in the fact that a selfish individual helps another at a cost to itself. Cost is also the byproduct of other prosocial behaviors, e.g., costly punishment ${ }^{41}$. Recently, Suzuki and Kimura reported that indirect reciprocity is sensitive to the costs of information transfer. Their work introduced the hypothesis that information transfer (or reputation building) is costly into indirect reciprocity models, making indirect reciprocal behaviors as costly as cooperative behaviors. Although indirect reciprocity helps explain cooperation among unrelated strangers, cost might be a handicap. To the best of my knowledge, the effects of the cost of reputation-based partner switching on cooperation have sel- dom been considered in previous partner-switching models. The present model extends previous ones primarily by considering this cost of partner-switching behaviors.

In different contexts, social dilemmas frequently involve a group of individuals. For example, say a number of players maintain a public good from which all members benefit unconditionally. In such a situation, a homo economicus should choose the defection strategy. Referred to as partnership adjustment in multiple-person games, a player chooses a group to join. In most spatial models of PGG, each agent hosts a game and takes part in games hosted by others. The group selection problem de facto becomes the individual selection problem. A homo economicus is likely to choose an individual who can maximize his personal welfare, whereas a homo reciprocans most likely chooses another counterpart. If the partner-switching behaviors are costly, the evolution of such costly behaviors becomes as problematic as the evolution of costly cooperative behaviors. In this model, I do not presume that the partner-switching behavior of an individual is unchanging during the game's evolution. Partnerswitching behavior can be imitated or learned in the same way as game-strategic behavior. Using numerical simulations, I show that a mechanism of reputation evaluation that praises both cooperative and partner-switching behaviors allows them to coevolve.

\section{Model}

Strategic behaviors. The present model describes the evolution of genetically transmitted behavioral types in a population of semirational or rational agents who engage in game interactions and partnership adjustments. There are two classes of individual behaviors regarding partner switching and game playing. The particular behavior of an agent is determined by his preferences. Two opposite preferences, i.e., C and D, condition agents' behavioral choices in game playing. Agents can adjust their partnerships under a reputationmaximization rule $(\mathrm{R})$ or do nothing $(\mathrm{N})$. Such a partner-switching rule enables an agent to switch from the lowest-reputation neighbor to the highest-reputation one among their next-nearest neighbors. Therefore, agents' behaviors are represented as the expression of two hypothetical alleles at each of two loci. CR agents cooperate in the period of game playing and adjust their partnerships based upon reputation during partner switching. In the same manner, the behaviors of DR, CN, and DN agents are determined. For the sake of mathematical expression, agent $i$ 's strategy $s_{i}$ is denoted as a twodimensional binary vector $\left[s_{i 1}, s_{i 2}\right]$. The first dimension expresses the cooperative preference, and the second dimension expresses the preference for partner switching. To be more specific, $s_{i 1}=1$ means that agent $i$ is cooperative, whereas $s_{i 1}=0$ means that agent $i$ exhibits the opposite preference. For partner switching, $s_{i 2}$ $=1$ means that agent $i$ is willing to adjust his partnership if necessary and pay the cost associated with such a behavior, whereas $s_{i 2}=0$ means that agent $i$ never makes any adjustment.

Game interactions. Agents autonomously organize into a number of groups in which members in the same group play a game together. Their interaction patterns, i.e., who interacts with whom, are defined by a dynamical network. Agents are placed on the nodes, which are connected by links. Each agent can sponsor a game in which his linked neighbors can participate. In a game sponsored by agent $i$, agent $j$ obtains a payoff $p_{s_{j}}(i)$ if he has a link to $i$ and thus plays as a group member. The evolutionary fitness of an agent is the accumulated payoffs obtained from all the games that he is involved in, i.e.,

$$
f(i)=p_{s_{i}}(i)+\sum_{j \in \mathcal{N}_{i}^{1}} p_{s_{i}}(j),
$$

where $\mathcal{N}_{i}^{1}$ is the set of linked neighbors of agent $i$. 
I resort to a PGG as a metaphor to model the interactions among group members. PGGs describe a situation where the collective benefit is shared among the members in the group. In a typical PGG, cooperators contribute a fixed amount of effort $c$ to the common pool. The amount of the overall contributions is amplified by a factor $\alpha$ and shared among all group members. In a PGG sponsored by agent $i$, agent $j$ obtains a payoff as a group member according to the following:

$$
p_{s_{j}(i)}= \begin{cases}\frac{\alpha n_{C}(i) c}{\left|\mathcal{N}_{i}^{1}\right|+1} & \text { if } s_{j 1}=0 \\ \frac{\alpha n_{C}(i) c}{\left|\mathcal{N}_{i}^{1}\right|+1}-c & \text { else. }\end{cases}
$$

In this function, $n_{C}(i)$ is defined as the number of cooperative agents in group $i$. If agent $j$ is a cooperator, i.e., $s_{j 1}=1$, then $n_{C}(i)$ is increased by $1 .\left|\mathcal{N}_{i}^{1}\right|$ is the number of agent $i$ 's linked neighbors.

Coevolutionary dynamics. Agents engage in the two coupled evolutionary processes of partnership adjustment and strategy update. At each time step of the coevolution, a random agent adjusts his partnership with probability $\mathrm{Pr}$ or otherwise updates his strategy by imitation. The dynamics of strategy update is inherited directly from previous models; namely, smoothed imitation dynamics is adopted ${ }^{36,42}$. The probability that agent $i$ successfully adopts the strategy of one of his neighbor $j$ is

$$
\omega\left(s_{i} \leftarrow s_{j}\right)=\frac{1}{1+\exp [-(f(j)-f(i)) / \theta]},
$$

where agent $j\left(j \in \mathcal{N}_{i}^{1}\right)$ is chosen randomly and $\theta$ is the noise extent. The two alleles of $j$ are transmitted to $i$ as a whole if the imitation occurs; thereafter, $s_{i 1}=s_{j 1}$ and $s_{i 2}=s_{j 2}$.

Partner-switching behaviors. In agreement with previous studies, individuals adjust their partnerships by choosing the candidate with the maximal reputation. The minimum information required for a partner-switching agent is the accurate reputations of his nearest neighbors and next-nearest neighbors. The renewal of each agent's reputation score occurs at every time step according to the following equation $^{36}$ :

$$
k_{i}(t)=\lambda k_{i}(t-1)+s_{i 1},
$$

where $\lambda$ is the discounting rate of reputation. The score of an individual's reputation is his previous score plus the performance of the game-strategic behavior in the current time step.

This model introduces four strategies regarding cooperation and partnership adjustment. I propose an improved reputation evaluation function that further values partner-switching behavior. As a simple improvement on Eq. 4, an individual's game-strategic and partner-switching behaviors can be weighted equally:

$$
k_{i}(t)=\lambda k_{i}(t-1)+s_{i 1}+s_{i 2} .
$$

When agent $i\left(s_{i 2}=1\right)$ modifies his partnership, he severs the neighbor who has the minimum reputation among all his nearest neighbors: $\arg \min \left(k_{j}(t)\right)\left(j \in \mathcal{N}_{i}^{1}\right)$. Then, he chooses as a new partner the individual who has the maximum reputation among all his nextnearest neighbors: $\arg \max \left(k_{j}(t)\right)\left(j \in \mathcal{N}_{i}^{2}\right)$. In a model of friendship formation $^{43}$, an agent incurs an opportunity cost regardless of the outcome for each unit of time in the matching process. In this model, the severed agent is the one with the minimum reputation, who may play as an exploiter in the PGG hosted by the focal agent. Therefore, the focal agent has an incentive to adjust the partnership even at a cost to himself. Moreover, a reputation system incurs more costs, e.g., the cost of information dissemination and maintenance and the cost of reputation evaluation. I assume that these costs are shared by all the agents involved in this process. Specifically, each agent who exhibits the $R$ trait has to pay a certain $\operatorname{cost} c_{R}$ :

$$
f^{\prime}(i)=f(i)-c_{R} \text { if } s_{i 2}=1 .
$$

In a reputation system where game-strategic behaviors are considered, the second-order assessment also depends on the score of the receiver'; for example, it can be deemed bad to help a bad person. The partner-switching activity defined here will possibly increase the overall welfare of the public goods hosted by the focal agent. The $R$ trait of an agent also implies that he accepts his responsibility to share the cost during the partner-switching process. For others, or at least linked neighbors, the partner-switching behavior of the focal agent can be deemed a prosocial behavior. It is therefore assumed that a partner-switching behavior raises the focal agent's reputation, which is conveyed in Eq. 5.

\section{Results}

I conducted numerical simulations using a wide range of parameters. To quantify the equilibrium frequencies of strategies, the average fraction of a strategy was sampled during the last 1000 steps after sufficient Monte Carlo steps (MCSs). Under the same parameter setting, data were further averaged over 32 independent simulations. $\rho_{C}$ is the equilibrium frequency of strategy C, whereas $\rho_{R}$ is the equilibrium frequency of strategy $\mathrm{R}$. Due to the disconnection of interactions, some agents are isolated without any connections to others. These independent agents were excluded in the computation of strategy frequencies. Without specification, the initial population consisted of 2500 agents whose strategies were random, and the initial interaction network was a periodical square lattice $(\langle d\rangle=4)$. Following common practice, a middle level of imitation noise $(\theta=$ 0.1 ) was set. The default setting of the discounting rate of reputation is 0.5 .

Phase diagrams of the equilibrium frequencies of the behaviors. In all simulations, $c=1$. Therefore, the partner-switching behavior presumably falls within the range of $0 \leq c_{R} \leq 1$. When $c_{R}=1$, the partner-switching behavior becomes as costly as the cooperative behavior. Previous literature has shown that the average vertex degree $\langle d\rangle$ is an important factor for the evolution of cooperation in networked games ${ }^{44}$. Here, I adopt a normalized amplification factor of PGG $\eta=\alpha /(\langle d\rangle+1)$. In Fig. 1, the simulation results are shown under two sets of simulations. The top panel shows the situation at a low partner-switching probability $(\operatorname{Pr}=0.2)$, whereas the bottom shows the situation with the same parameter values as the top panel except $P r=0.8$.

In Fig. 1(a), $\rho_{C}$ increases with increasing $\eta$. A higher level of $c_{R}$ increases the critical value $\eta_{C}{ }_{C}$ for $\eta$, above which cooperators coexist with defectors. However, such an effect is weak. In Fig. 1(b), the phase of $\rho_{R}$ exhibits a nonlinear transition. $\rho_{R}$ does not always increase with increasing $\eta$. On the whole parameter plane, $\rho_{R}$ does not reach $70 \%$. In the parameter range of $0 \leq \eta \leq 0.6, \rho_{R}$ decreases sharply to 0 with increasing $c_{R}$; that is, the reputation-based partner-switching behavior is sensitive to its cost. This result corresponds with the work of Suzuki and Kimura. However, the decrease in $\rho_{R}$ becomes gentler at higher levels of $\eta$. Let us analyze the situation at $P r=0.8$. The critical value $\eta_{C}^{\prime}$ for the emergence of cooperation is lowered remarkably. In Fig. 1(d), the nonlinear phenomenon is more obvious than that in Fig. 1(b). In the phase where $\rho_{C}$ experiences a transition, $\rho_{R}$ reaches the maximum. In other words, the evolution of cooperation and the evolution of reputation-based partnership adjustment might be correlated.

Coevolution under the improved reputation function. Compared with Eq. 4, the improved function of reputation (Eq. 5) can evaluate individuals' behaviors with respect to both game playing and partner 
(a)

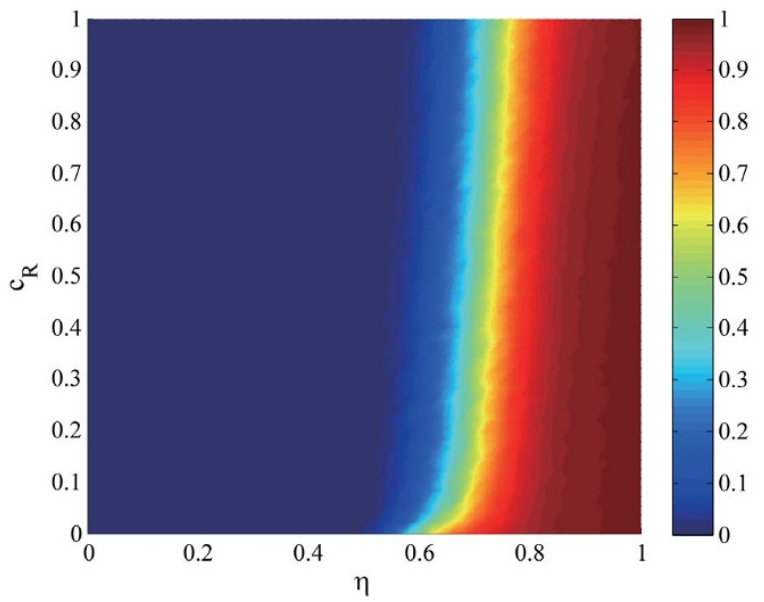

(c)

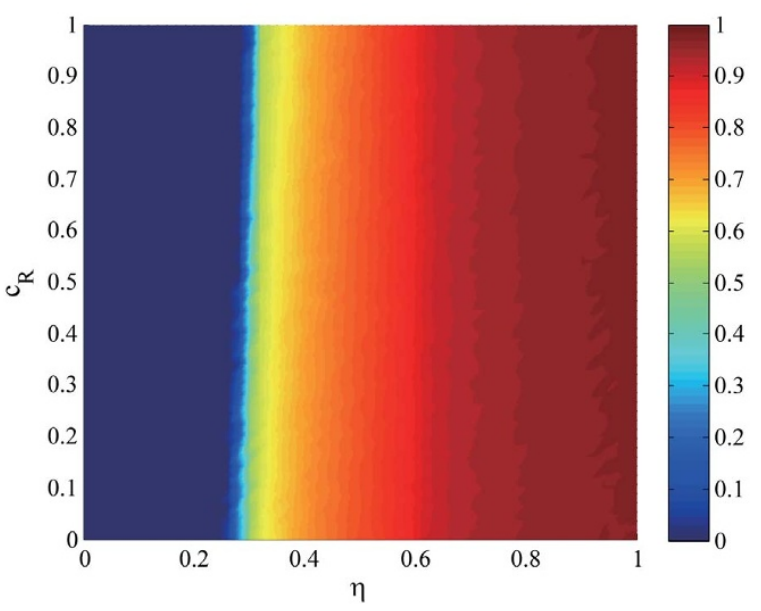

(b)

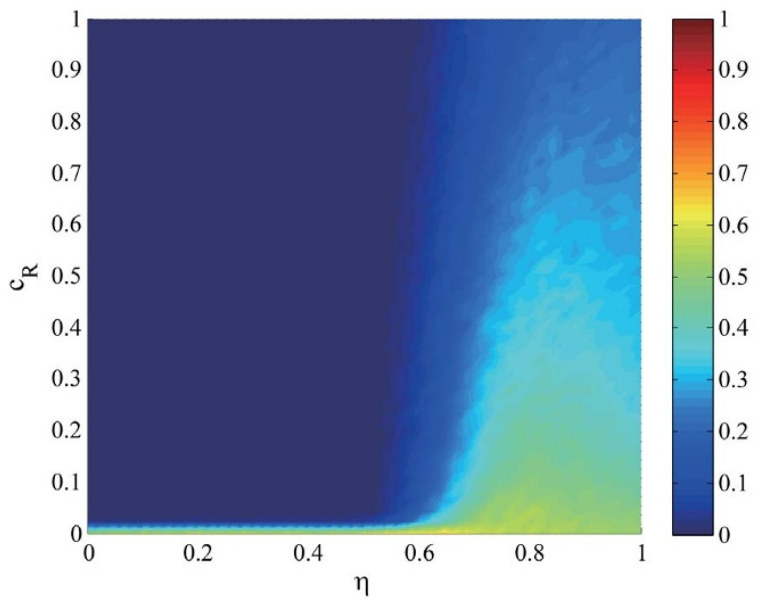

(d)

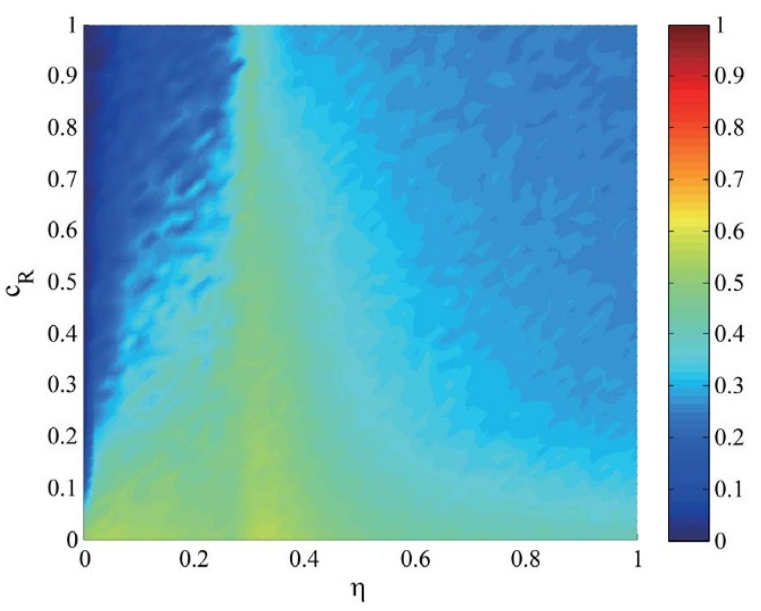

Figure $1 \mid$ The effects of the cost of partner switching on equilibrium strategy frequencies. Top panel: the partner-switching probability $P r=0.2$; bottom panel: $\operatorname{Pr}=0.8$. In each panel, the frequency of cooperative strategy $\rho_{C}$ and the frequency of reputation-based partner-switching strategy $\rho_{R}$ are shown as a function of both the normalized amplification factor and the cost of partner switching $\left(\eta, c_{R}\right)$, respectively. In (b), $\rho_{R}$ decreases to 0 rapidly with increasing $c_{R}$ in the parameter range of $0 \leq \eta \leq 0.6$. When $\eta$ becomes larger, the evolution of partner-switching behavior is facilitated. In (d), there is no phase where $\rho_{R}$ rapidly decreases to 0 with increasing $c_{R}$.

switching. Fig. 2 shows the equilibrium frequencies in the situation under Eq. 5. Figs. 2 and 1 can be compared directly because they share the same parameter and simulation settings. Fig. 2(a) looks quite similar to Fig. 1(a). In Fig. 2(c), $\rho_{C}$ experiences a slower transition from 0 to the maximum. The phase diagrams of partner-switching behavior under the improved rule display more interesting results. $\rho_{R}$ is much higher in Fig. 2(b) than in Fig. 1(b). In Fig. 2(d), $\rho_{R}$ is increased on average as well. The enhancement of the frequencies of partner-switching behavior can be attributed to the improvement of reputation evaluation. In Fig. $2(\mathrm{~d}), \rho_{R}$ does not vary significantly with the variation of $c_{R}$. The influence of the cost associated with partner-switching behavior is trivial when agents can perform such behaviors more frequently. Moreover, the correlation between cooperation and reputation-based partnership adjustment can also be observed. Before stepping into the next analysis, we can conclude that the prevalence of reputation-based partner-switching behavior is aided by some refined rules of reputation evaluation.

The phase diagrams of strategy frequencies with fixed normalized amplification factors of PGG are depicted in Fig. 3. Two cases of normalized amplification factors are studied: 0.7 and 0.9 . The former setting allows a phase transition of $\rho_{C}$, whereas the latter setting enables a high-level $\rho_{C}$. At the same level of $c_{R}, \rho_{C}$ does not increase monotonously with $P r$, and neither does $\rho_{R}$. Remember that a random agent reconnects to a new one with probability Pr or otherwise updates his strategy. Hence, an optimal partner-switching probabil- ity for the emergence of prosocial behaviors is likely to be a medium value. In the bottom panel, the optimal value of $\operatorname{Pr}$ shifts toward 0 . Empirical research has shown that intermediate levels of change in social ties lead to optimal levels of cooperation in a series of online experiments ${ }^{45}$. The simulation result of this model echoes the empirical finding.

As a complement to phase diagrams of the $\mathrm{C}$ and $\mathrm{R}$ traits, Fig. 4 shows equilibrium frequencies of the $\mathrm{CR}, \mathrm{CN}, \mathrm{DR}$ and $\mathrm{DN}$ strategies as a function of the cost of partner switching, the amplification factor and the partner-switching probability, respectively. Fig. 4(a) shows that, with increasing $c_{R}$, the equilibrium frequency of the CR strategy $\rho_{C R}$ decreases. Let us analyze the situations where the equilibrium frequencies of prosocial behaviors experience a phase transition from 0 to certain levels. In Fig. 4(b), $\rho_{C R}$ increases with increasing $\eta$. Meanwhile, $\rho_{D N}$ decreases with increasing $\eta$. When the social productivity is at a fixed level, as shown in Fig. 4(c), the equilibrium frequency of the CR strategy does not increase monotonously with the partner-switching probability. A high frequency of partnership adjustment might not lead to an optimal social status, which is in accord with the analysis of Fig. 3. In both Figs. 4(b) and (c), the CN strategy is promoted weakly. Therefore, the CR strategy has an evolutionary advantage over the $\mathrm{CN}$ strategy under the improved reputation function. Moreover, it can be seen that the DR strategy is suppressed in the coevolution.

Fig. 5 is devoted to investigating the effect of a high partnerswitching probability on the coevolution of cooperation and 
(a)

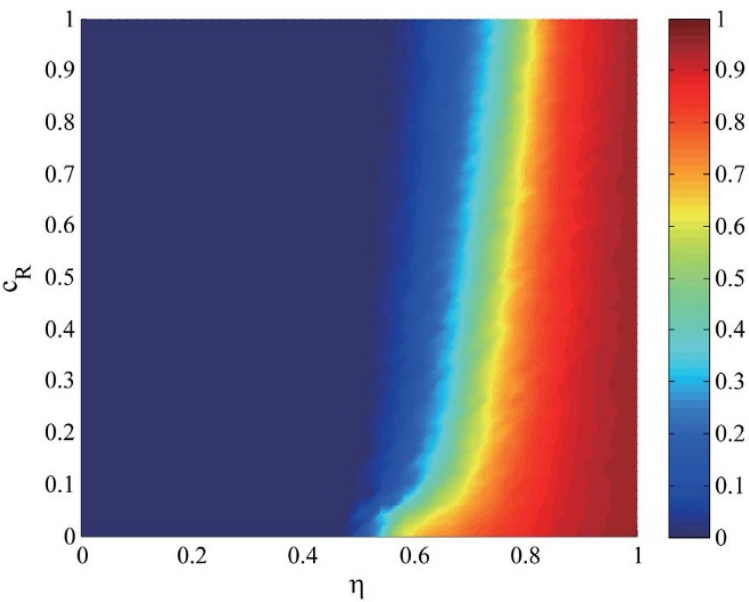

(c)

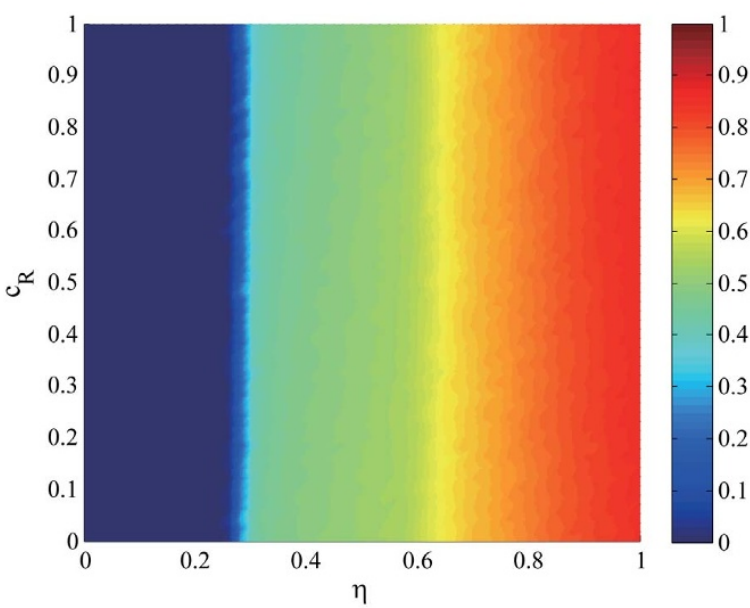

(b)

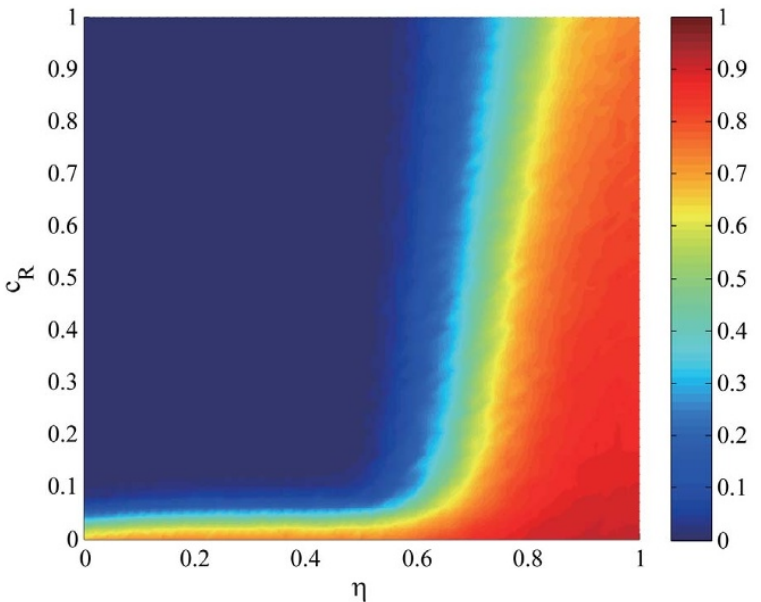

(d)

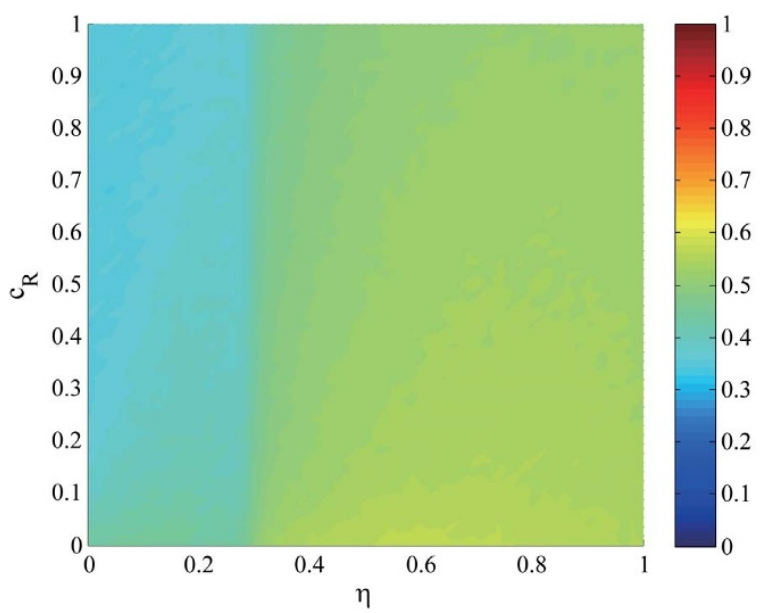

Figure $2 \mid$ The effects of the cost of partner switching on equilibrium strategy frequencies under an improved reputation evaluation rule as described in Eq. 5. Top panel: partner-switching probability $\operatorname{Pr}=0.2$; bottom panel: $\operatorname{Pr}=0.8$. In each panel, the frequency of cooperation $\rho_{C}$ and the frequency of reputation-based partnership adjustment $\rho_{R}$ as a function of both the normalized amplification factor and the cost of partner switching $\left(\eta, c_{R}\right)$ are presented, respectively. The improved rule takes the prosociality of reputation-based partner-switching behaviors into account in the evaluation of reputation. Compared with Fig. 1, such a rule enhances $\rho_{R}$ significantly.

reputation-based partnership adjustment. For visual convenience, a population consisting of 256 agents was set. The simulations were performed using high values for $c_{R}$ and $\eta$. The situations at $\operatorname{Pr}=0.2$ and $P r=0.8$ were compared. I sampled the detailed partnership networks and investigated the strong effect of partner switching over network structures. In Fig. 5, different agents are colored differently. Clearly, more separate subnetworks and isolated nodes were developed in the situation at $\operatorname{Pr}=0.8$. Agents that are segregated from the most highly connected subgraph have less of a chance to imitate prosocial agents. Therefore, a high-level partner-switching probability might not be good for the evolution of either cooperative or reputation-based partner-switching behaviors.

The investigation of reputation-based reciprocity. Indirect reciprocity is unique in that one individual has helped another and therefore is helped by a third one. Such a reciprocity pattern pivots on reputation, which is called "reputation-based reciprocity" or "downstream reciprocity". Whether the coevolution of prosocial behaviors in this model is ascribable to reputation-based reciprocity was further investigated. The formation of new links manifests the basic pattern of reputation-based reciprocity. Consider a new link reconnecting to a cooperator: the more likely a cooperator is at the other end, the stronger the effect of reputation-based reciprocity. A proper measure to quantify this effect in a period of evolutionary time is the ratio of the cooperator-cooperator links to the total number of links reconnecting to cooperators $\frac{l_{C C}}{l_{C C}+l_{D C}}$.

Two simulations were run with the same parameters except for different amplification factors, which resulted in two typical coevolution processes with distinct outcomes, as shown in Fig. 6. $\frac{l_{C C}}{l_{C C}+l_{D C}}$, $\rho_{C}$ and $\rho_{R}$ are displayed as a function of evolutionary time step, and the time window for sampling was 1000 MCSs. Fig. 6(a) displays an evolutionary process in which a prosocial regime emerged successfully, whereas Fig. 6(b) reports a negative example. $\frac{l_{C C}}{l_{C C}+l_{D C}}$ exhibits remarkably different levels in these two processes. When $\rho_{C}$ and $\rho_{R}$ experience a transition to high levels, as shown in Fig. 6(a), $\frac{l_{C C}}{l_{C C}+l_{D C}}$ exhibits high levels transiently. Additionally, there is a period of time when $\frac{l_{C C}}{l_{C C}+l_{D C}}$ is mostly $100 \%$. When the coevolution arrives at a stationary stage, there are no new links reconnecting to cooperators. This comparison analysis indicates that a high level of social productivity creates a niche for reputation-based reciprocity, which allows its functionality in the achievement of high frequencies of prosocial behaviors. Thus, the basic pattern of reputation-based reciprocity underlies the coevolution in this model. 
(a)

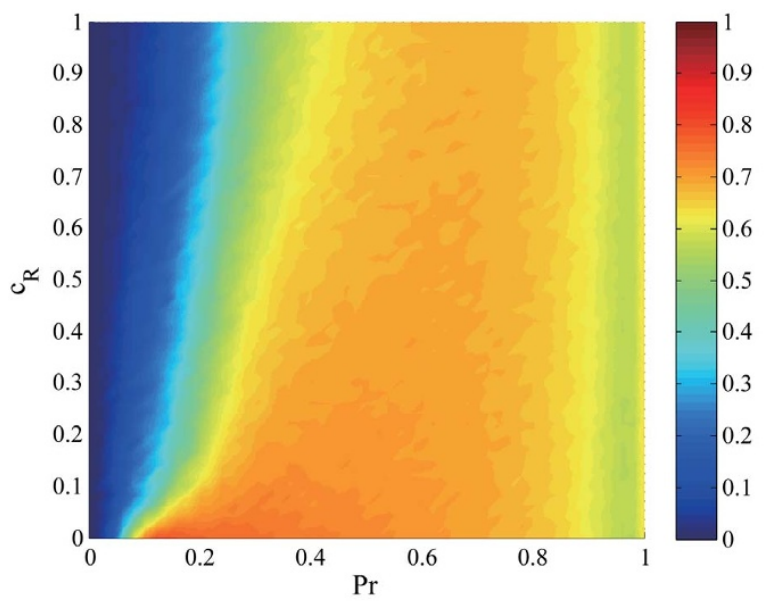

(c)

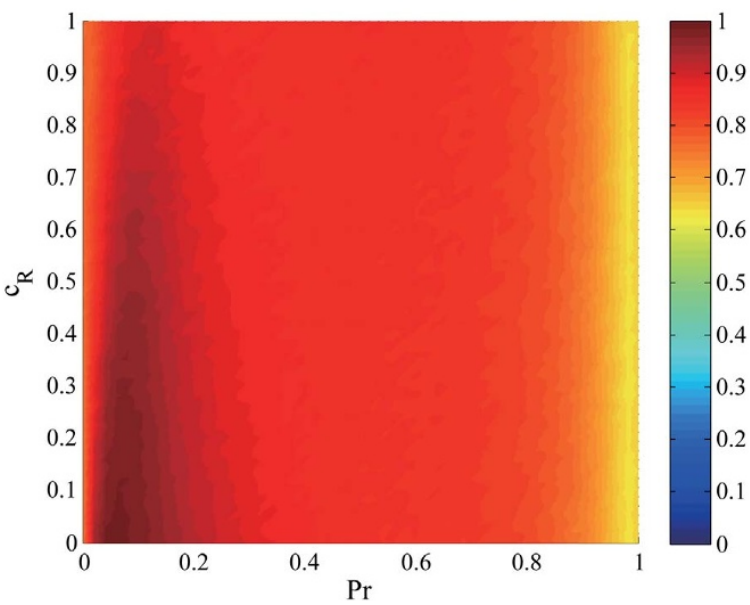

(b)

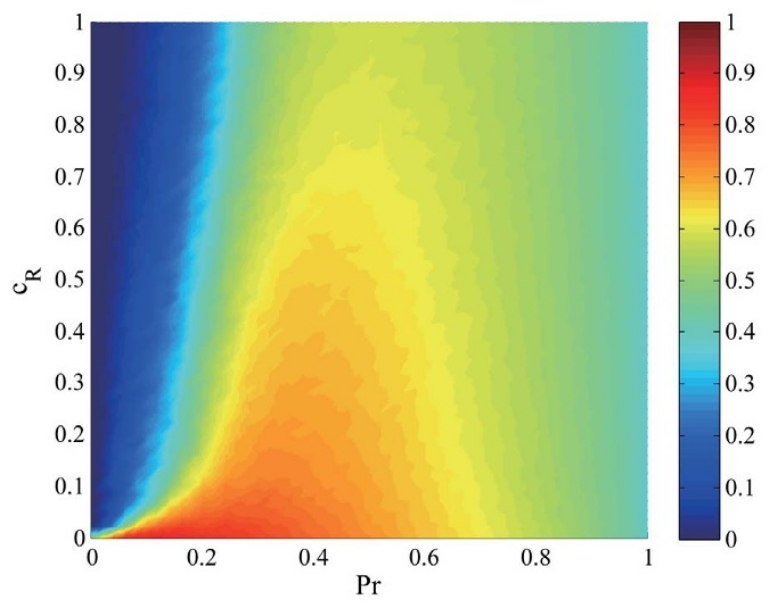

(d)

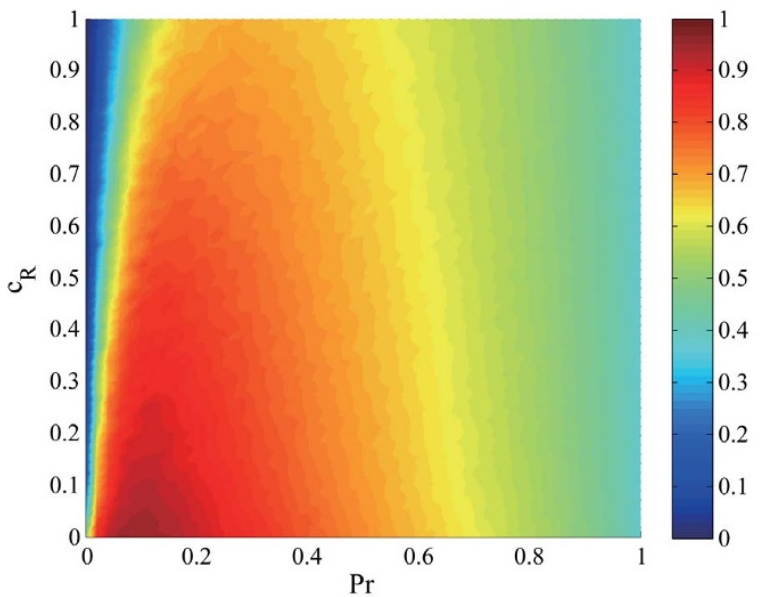

Figure $3 \mid$ The effects of the cost of partner switching on equilibrium strategy frequencies with fixed normalized amplification factors of PGG. Top panel: the normalized amplification factor $\eta=0.7$; bottom panel: $\eta=0.9$. In each panel, the equilibrium frequency of the cooperative behavior $\rho_{C}$ and the equilibrium frequency of the partner-switching behavior $\rho_{R}$ as a function of both the partner-switching probability and the cost of partner switching $\left(\operatorname{Pr}, c_{R}\right)$ are displayed, respectively. The average degree of the partnership network $\langle d\rangle=4$.

The effects of other factors. So far, I have investigated the principal factors that condition the coevolutionary dynamics under study. Finally, the effects of reputation decay and the average number of partners are examined. I report the equilibrium frequencies as a function of $c_{R}$ under different combinations of $\mathrm{Pr}$ and the discounting rate of reputation $\lambda$ in Fig. 7. Note that previous results were obtained from simulations at $\lambda=0.5$. When the partner-switching probability is small $(P r=0.2)$, different reputation decay rates have little influence on the equilibrium strategy frequencies. However, a full memory of previous reputation scores

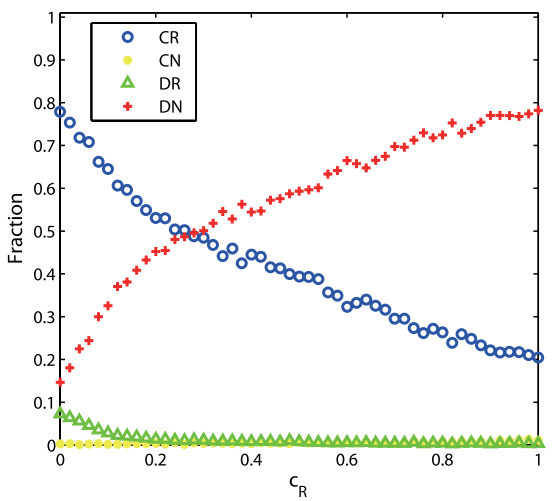

(a)

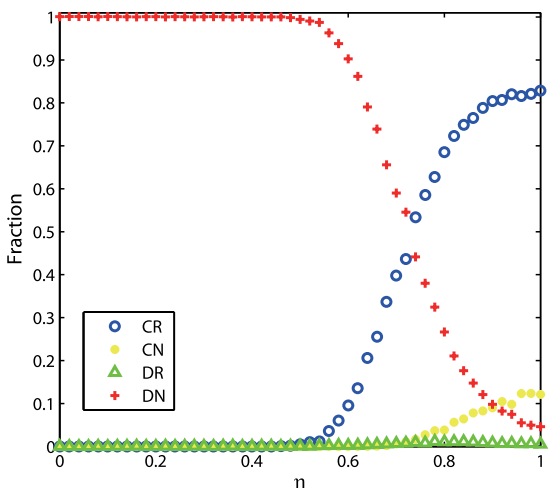

(b)

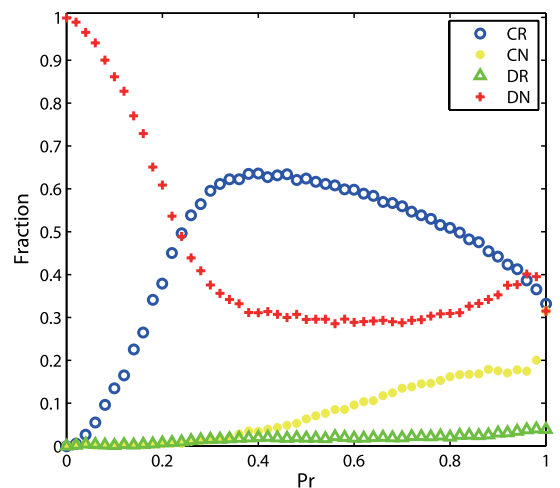

(c)

Figure $4 \mid$ The equilibrium frequencies of four strategies as a function of the cost of partner switching $c_{R}$, the normalized amplification factor $\eta$ and the partner-switching probability $P r$, respectively. (a) $\eta=0.7, \operatorname{Pr}=0.2$; (b) $c_{R}=0.5, \operatorname{Pr}=0.2$; (c) $c_{R}=0.5, \eta=0.7$. The data were sampled during the simulations for Figs. 2 and 3. In (a), $\rho_{C R}$ decreases with increasing $c_{R}$, whereas $\rho_{D N}$ increases with increasing $c_{R}$. Subfigures (b) and (c) show the situations where the cost of partner switching is fixed. At a given partner-switching probability, the CR strategy becomes the fittest as $\eta$ increases. With a fixed $\eta$, the highest frequency of CR occurs at a middle value of $P r$. 


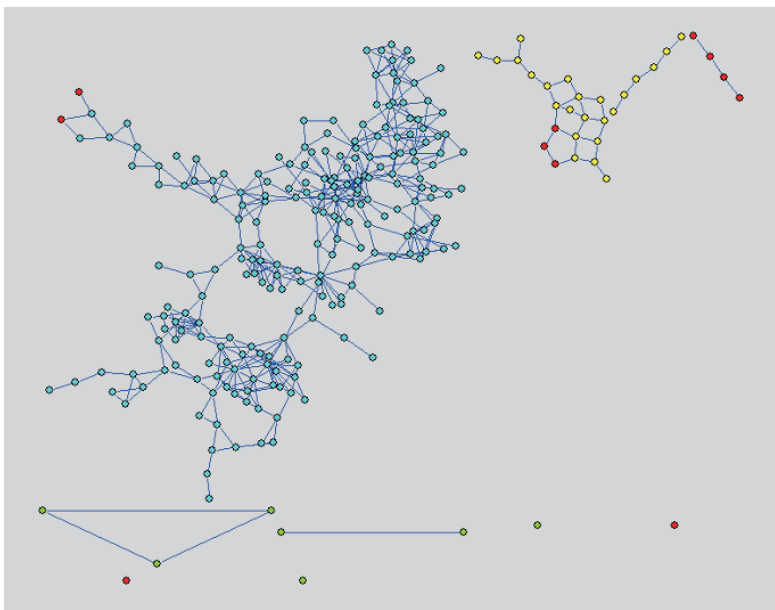

(a)

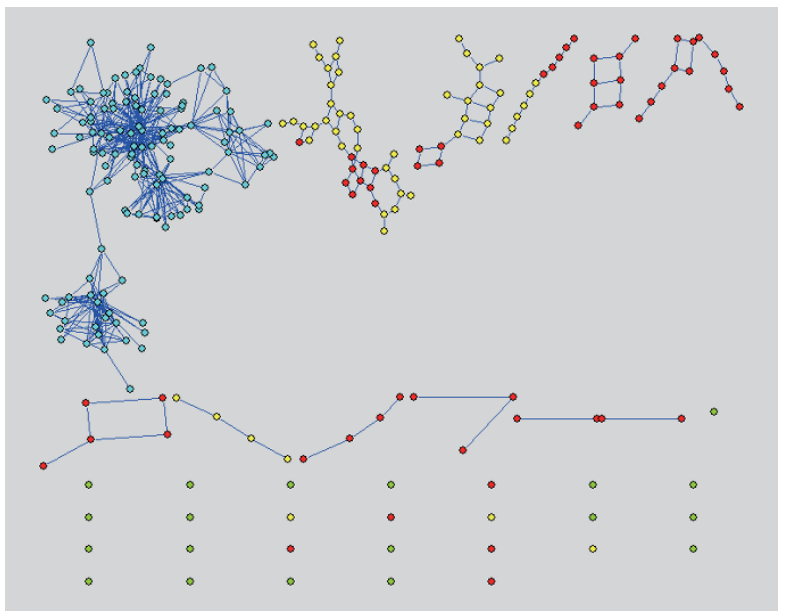

(b)

Figure 5 An analysis of the strong effect of partner switching on the coevolution of cooperation and reputation-based partnership adjustment. (a) An interaction network sampled when the population reached the stationary regime at partner-switching probability $\operatorname{Pr}=0.2$; (b) An interaction network sampled when the population reached the stationary regime at $\mathrm{Pr}=0.8$. Blue color denotes the CR strategy; yellow denotes the CN strategy; green denotes the DR strategy; and red denotes the DN strategy. The total number of agents is 256 . A high frequency of partner switching induces more separate subnetworks and isolated vertices. Other parameter values are the cost of partner switching $c_{R}=0.96$, the normalized amplification factor $\eta=0.98$ and the average degree of the partnership network $\langle d\rangle=4$. These pictures were drawn in Pajek ${ }^{54}$.

$(\lambda=1)$ affects the coevolution when the partner-switching probability increases. At $\operatorname{Pr}=0.4,0.8$, the trajectories for $\lambda=1$ are lower than the trajectories for $\lambda=0$ or 0.5 .

The simulation result with $\langle d\rangle=8$ is displayed in Fig. 8 . The parameters are the same as those of the top panel of Fig. 2. Remarkably, both the critical value of $\eta$ for cooperation and the critical value of $\eta$ for partner switching are lowered. Additionally, the transitional phases of both $\rho_{C}$ and $\rho_{R}$ from 0 to high levels become narrower compared with Fig. 2. When interaction patterns are fixed, a higher average vertex degree usually makes the evolution of cooperation less likely. However, it is much different in situations where agents dynamically adjust their social relationships.

\section{Discussion and Conclusions}

Reputation can steer individuals' practice of partnership adjustment, resulting in a selection force against defectors. Fu et al. proposed a

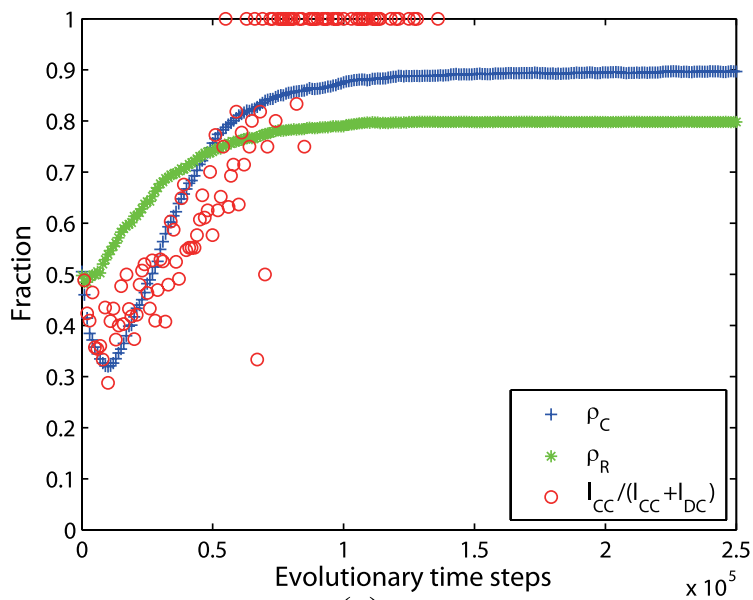

(a) model that describes the coevolution of game playing and partner switching $^{36}$. At each time step, a random individual can either alter his strategy by imitating his nearest partners or adjusts his partnerships by severing the tie to the neighbor with the lowest reputation and reconnecting to a new partner. They found that reconnecting to the next-nearest neighbor who has the highest reputation is more beneficial to cooperation than random adjustment. Notwithstanding the inheritance of reputation-based partnership adjustment, this model advances on previous ones in two key respects. First, the individual's partner-switching behavior is governed by an allele and is heritable in this model; namely, an agent does not have a fixed partner-switching preference during his evolutionary life. Second, individuals who are willing to adjust their partnerships have to pay a cost for evaluating reputation and broadcasting information. These assumptions allow us to investigate the conditions for the evolution of costly partner-switching behaviors in a reputation system. By

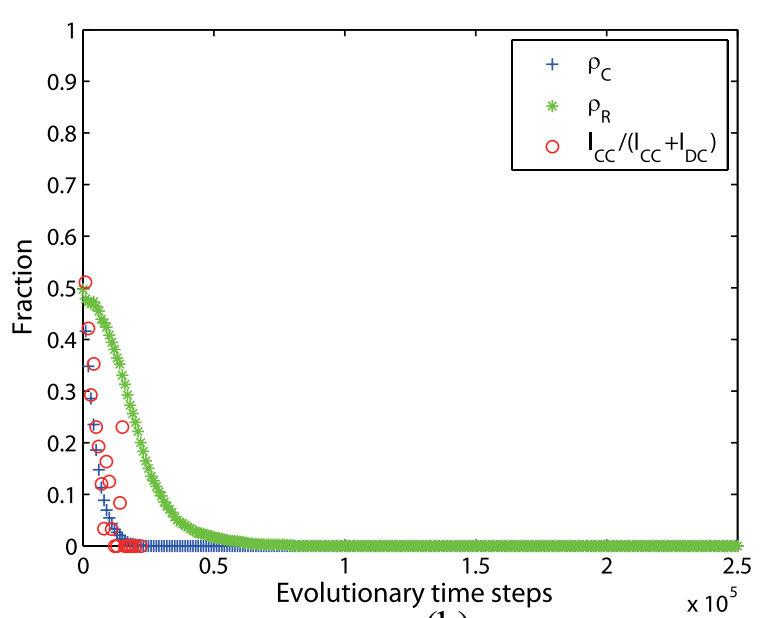

(b)

Figure 6 | The manifestation of the basic pattern of reputation-based reciprocity. Two parallel simulations were sampled: (a) A high level of reputation-based reciprocal effects allows the prevalence of prosocial behaviors (the normalized amplification factor $\eta=0.9$ ); (b) A low level of reputation-based reciprocal effects leads to the extinction of prosocial behaviors $(\eta=0.5)$. The basic pattern of reputation-based reciprocity is rooted in the formation of new links. The level of reputation-based reciprocity is measured by the ratio of the cooperator-cooperator links to the total number of links reconnecting to cooperators, $\frac{l_{C C}}{l_{C C}+l_{D C}}$, in a period of evolutionary time. For both cases, other parameters are the partner-switching probability $\operatorname{Pr}=0.2$ and the cost of partner switching $c_{R}=0.5$. 


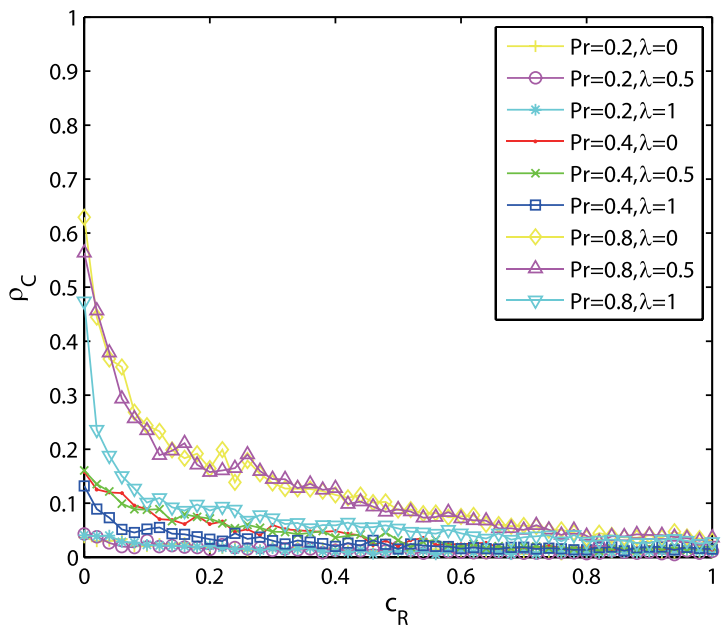

(a)

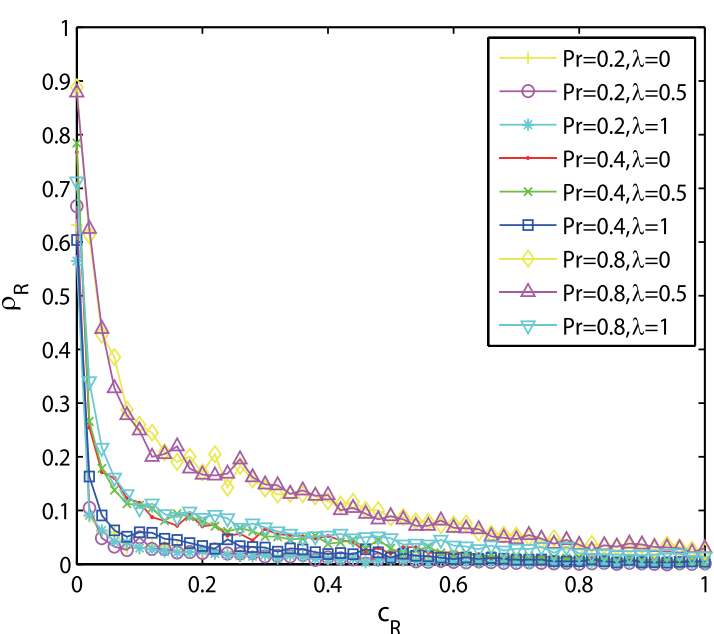

(a)

Figure $7 \mid$ The effects of the discounting of reputation score on the coevolution of cooperation and reputation-based partnership adjustment.

(a) The $\mathrm{C}$ frequency $\rho_{C}$ as a function of the cost of partner switching $c_{R}$ under different combinations of the partner-switching probability $\operatorname{Pr}$ and the discounting rate of reputation $\lambda$; (b) The R frequency $\rho_{R}$ as a function of $c_{R}$ under different combinations of $\operatorname{Pr}$ and $\lambda$. As shown, the discounting of the reputation score has a weak influence over the strategy frequencies. The normalized amplification factor $\eta=0.7$.

(a)

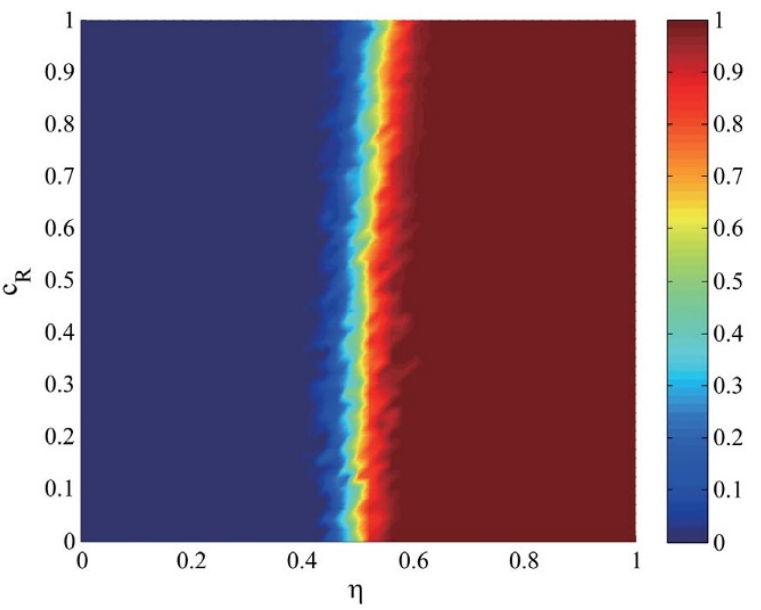

(c)

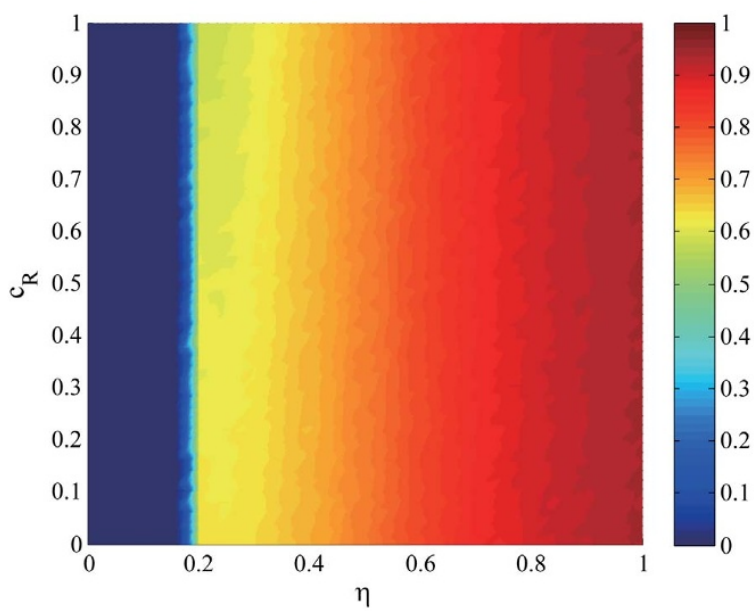

(b)

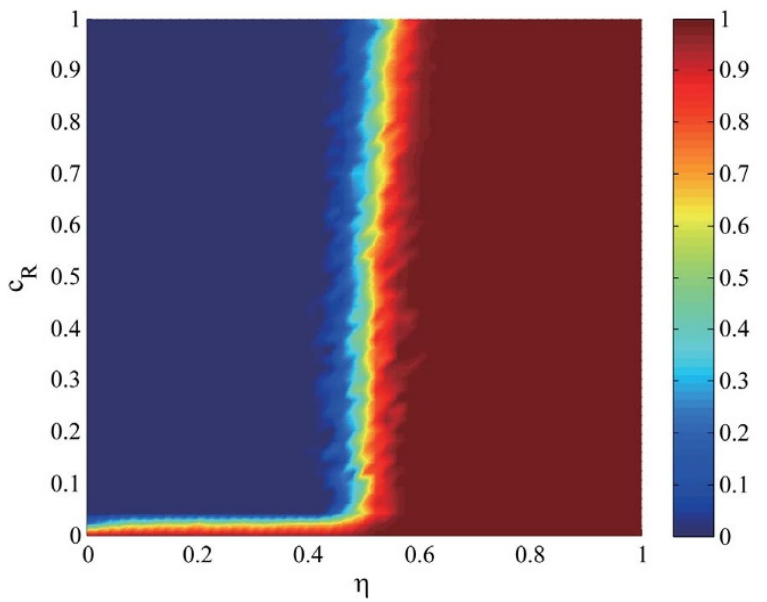

(d)

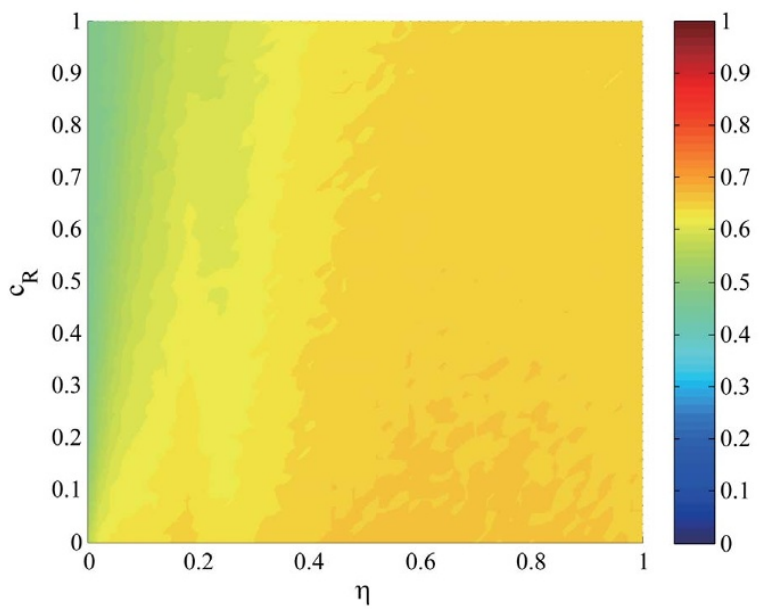

Figure $8 \mid$ Phase diagrams of the equilibrium strategy frequencies when agents have more partners on average. The initial population structure was a square lattice with a Moore neighborhood, i.e., the average degree of the partnership network $\langle d\rangle=8$. Top panel: the partner-switching probability $\operatorname{Pr}=$ 0.2; bottom panel: $\operatorname{Pr}=0.8$. In each panel, the $\mathrm{C}$ frequency $\rho_{C}$ and the R frequency $\rho_{R}$ as a function of both the normalized amplification factor and the cost of partner switching $\left(\eta, c_{R}\right)$ are presented, respectively. Other parameters are the same as those of Fig. 2. Comparing Fig. 8(b) with Fig. 2(b), the parameter range of $\eta$ where $\rho_{R}$ decreases rapidly with increasing $c_{R}$ becomes narrower. 
numeric simulations, I obtained two-dimensional phase diagrams for game-strategic behaviors and partner-switching behaviors in a wide parameter range. I show that partner-switching behaviors can evolve even in the presence of a certain cost. There is a concurrence between the evolution of cooperative behaviors and the evolution of partner-switching behaviors, implying a mutual enhancement effect between these two prosocial behaviors.

In an analytical model, Suzuki and Kimura showed that selfish individuals never build a reputation and that indirect reciprocity fails once reputation building is costly ${ }^{18}$. At a high level, the present results are consistent with the prior work in that the emergence and sustenance of reputation-based partner-switching behaviors are sensitive to their costs within a certain parameter range. However, such a handicap can be overcome with a large amplification factor of social productivity or a high probability of partner switching. Moreover, an improved rule of reputation evaluation counting the score of the partner-switching behavior significantly promotes its evolution, which correspondingly facilitates the evolution of cooperation. This result highlights the importance of reputation design in solving free-riding in other prosocial behaviors. In real societies, the architects of a social system should become aware of the power of policy design, which can channel the individual's interest toward the achievement of prosociality. Notably, the partner-switching models are different from typical models of indirect reciprocity in that there is an underlying interaction structure. Although the interaction network is dynamic with respect to a large time scale, agents transiently have relatively fixed partnerships. As is discussed in Suzuki and Kimura's article, network reciprocity may support the evolution of cognitive abilities to build reputations and thus indirect reciprocity. I show that in a dynamical social network, partner-switching behaviors based on reputation can evolve regardless of their costs.

More generally, these results might be extended to the case of assortative matching or homophily, i.e., where individuals with similar genotypes, phenotypes or strategies are more likely to interact with each other ${ }^{46,47}$. Assortative partner choice promotes cooperation in human experiments adopting both a $\mathrm{PGG}^{48,49}$ and a prisoner's dilemma game $e^{50}$. When humans can choose the subjects with whom they wish to interact, partner choice creates the possibility of altruism provided that individuals compete for good partners ${ }^{51}$. In fact, the partner choice theories have been tested in some biological environments, e.g., cleaner mutualism among fish ${ }^{52}$. A theoretical model reveals that homophily can evolve under a wide variety of conditions $^{47}$ and a genome-wide analysis of correlation in genotypes between human friends suggests that homophily may yield an evolutionary advantage $\mathrm{e}^{53}$. Although the cost is a byproduct of the behavior of assortative matching or homophily, those self-evolved agents might develop an ability to distinguish their counterparts. Nevertheless, this model is primitive, and some interesting refinements could be made. For example, partner-switching behavior is defined here as a binary value. A more realistic assumption is that an agent exhibits this behavior with a probability that is a continuous value and endogenously determined based on the agent's own interest. Furthermore, harnessing the free riding of public goods under stricter conditions remains to be explored. It is pivotal that a cooperator can end the mutual relationship between himself and a defector in this model. In the case of nonexcludable public goods, however, one cannot exclude others' participation in his game; an individual can merely adjust his own participation ${ }^{40}$. Once a cost is associated with such partner-switching behaviors, a reputation mechanism might be redesigned, or some other mechanisms may play a role as a complement.

The building blocks of humanity are a variety of prosocial behaviors with a prominent feature of cost. I show that a regime where cooperative and partner-switching behaviors persist collectively can be sustained on the conditions that reputation mechanisms are well designed and that some parameters are tuned. This work is beneficial to understanding the conundrum that humans are willing to involve in costly but prosocial behaviors regarding reputations.

\section{Methods}

As mentioned above, the analysis is based on a coevolution model of cooperation and partnership adjustment. Each agent has two hypothetical alleles which determine his behaviors for game playing and partner switching. In the initial stage of a simulation, agents are assigned with random allelic types and the partnership network is a square lattice with periodic boundary conditions. Each agent can host a PGG in which his linked neighbors can participate. Agents adjust their partnerships or update their strategies asynchronously in a randomized sequential order. The simulation algorithm consists of the following phases:

Phase 1 (Initialization). A population is generated and the simulation parameters are initialized.

Phase 2 (Coevolution). Steps 2.1-2.3, which constitute a complete MCS, are repeated until the coevolution reaches a steady state.

Step 2.1 (Game interaction). The fitness of each agent is set to zero. Each agent plays PGGs with its linked neighbors and obtains payoffs.

Step 2.2 (Reputation evaluation). Each agent's reputation score is renewed according to Eq. 4 or 5. Each agent who exhibits the R trait has to pay a certain cost.

Step 2.3 (Update dynamics). A random agent adjusts his partnership with a certain probability (Action 2.3.A) or otherwise updates his strategy (Action 2.3.B).

Action 2.3.A. The focal agent severs the tie to the neighbor with the lowest reputation and reconnects to the next-nearest neighbor who has the highest reputation if he exhibits the $\mathrm{R}$ trait.

Action 2.3.B. The focal agent changes his strategy by imitating one of his neighbors under a probability function, i.e., Eq. 3 .

The equilibrium frequencies of strategies for one simulation run result from averaging over $10^{3}$ MCSs after a transient period of more than $10^{5} \mathrm{MCSs}$. This procedure was repeated 32 times to produce each data point in Figs 1, 2, 3, 4, 7, and 8.

1. Hardin, G. The tragedy of the commons. Science 162, 1243-1248 (1968).

2. Axelrod, R. \& Hamilton, W. D. The evolution of cooperation. Science 211 1390-1396 (1981).

3. Dietz, T., Ostrom, E. \& Stern, P. The struggle to govern the commons. Science 302, 1907-1912 (2003).

4. Fehr, E. \& Fischbacher, U. The nature of human altruism. Nature 425, 785-791 (2003).

5. Nowak, M. A. Five rules for the evolution of cooperation. Science 314, 1560-1563 (2006).

6. Bowles, S. \& Gintis, H. Homo reciprocans. Nature 415, 125-128 (2002).

7. Russell, Y. I., Call, J. \& Dunbar, R. I. M. Image scoring in great apes. Behav Process 78, 108-111 (2008)

8. Engelmann, J. M., Herrmann, E. \& Tomasello, M. Five-Year Olds, but Not Chimpanzees, Attempt to Manage Their Reputations. PLoS ONE 7, e48433 (2012).

9. Nowak, M. A. \& Sigmund, K. Evolution of indirect reciprocity. Nature 437, 1291-1298 (2005).

10. Barclay, P. Reputational benefits for altruistic punishment. Evol Hum Behav 27, 325-344 (2006)

11. Sommerfeld, R. D., Krambeck, H.-J., Semmann, D. \& Milinski, M. Gossip as an alternative for direct observation in games of indirect reciprocity. Proc Natl Acad Sci 104, 17435-17440 (2007).

12. Nowak, M. A. \& Sigmund, K. Evolution of indirect reciprocity by image scoring Nature 393, 573-577 (1998).

13. Ohtsuki, H. \& Iwasa, Y. How should we define goodness?-reputation dynamics in indirect reciprocity. J Theor Biol 231, 107-120 (2004).

14. Ohtsuki, H. \& Iwasa, Y. The leading eight: Social norms that can maintain cooperation by indirect reciprocity. J Thoer Biol 239, 435-444 (2006).

15. Rockenbach, B. \& Milinski, M. The efficient interaction of indirect reciprocity and costly punishment. Nature 444, 718-723 (2006).

16. Ohtsuki, H., Iwasa, Y. \& Nowak, M. A. Indirect reciprocity provides only a narrow margin of efficiency for costly punishment. Nature 457, 79-82 (2009).

17. Lyle III, H. F. \& Smith, E. A. The reputational and social network benefits of prosociality in an Andean community. Proc Natl Acad Sci 111, 4820-4825 (2014).

18. Suzuki, S. \& Kimura, H. Indirect reciprocity is sensitive to costs of information transfer. Sci Rep 3, 1435 (2013).

19. Yoeli, E., Hoffman, M., Rand, D. G. \& Nowak, M. A. Powering Up with Indirect Reciprocity in a Large-scale Field Experiment. Proc Natl Acad Sci, 110, 10424-10429 (2013).

20. Camera, G., Casari, M. \& Bigoni, M. Money and trust among strangers. Proc Natl Acad Sci 110, 14889-14893 (2013).

21. Nowak, M. A. \& May, R. M. Evolutionary games and spatial chaos. Nature 359, 826-829 (1992).

22. Szabó, G. \& Fáth, G. Evolutionary games on graphs. Phys Rep 446, 97-216 (2007)

23. Brandt, H., Hauert, C. \& Sigmund, K. Punishment and reputation in spatial public goods games. Proc R Soc Lond B 270, 1099-1104 (2003).

24. Wang, X., Chen, X., Gao, J. \& Wang, L. Reputation-based mutual selection rule promotes cooperation in spatial threshold public goods games. Chaos, Solitons \& Fractals 56, 181-187 (2013). 
25. Zimmermann, M. G., Eguiluz, V. M. \& San Miguel, M. Coevolution of dynamical states and interactions in dynamic networks. Phys Rev E 69, 065102(R) (2004).

26. Pacheco, J. M., Traulsen, A. \& Nowak, M. A. Coevolution of Strategy and Structure in Complex Networks with Dynamical Linking. Phys Rev Lett 97, 258103 (2006).

27. Szolnoki, A. \& Perc, M. Emergence of multilevel selection in the prisoner's dilemma game on coevolving random networks. New J Phys 11, 093033 (2009).

28. Rand, D. G., Arbesman, S. \& Christakis, N. A. Dynamic social networks promote cooperation in experiments with humans. Proc Natl Acad Sci 108, 19193-19198 (2011).

29. Lee, S., Holme, P. \& Wu, Z.-X. Emergent Hierarchical Structures in Multiadaptive Games. Phys Rev Lett 106, 028702 (2011).

30. Pinheiro, F. L., Pacheco, J. M. \& Santos, F. C. From Local to Global Dilemmas in Social Networks. PLoS ONE 7, e32114 (2012).

31. Santos, M. D., Pinheiro, F. L., Santos, F. C. \& Pacheco, J. M. Dynamics of N-person snowdrift games in structured populations. J Theor Biol 315, 81-86 (2012).

32. Wang, J., Suri, S. \& Watts, D. J. Cooperation and assortativity with dynamic partner updating. Proc Natl Acad Sci 109, 14363-14368 (2012).

33. Barclay, P. Strategies for cooperation in biological markets, especially for humans. Evol Hum Behav 34, 164-175 (2013).

34. Tanimoto, J. Difference of reciprocity effect in two coevolutionary models of presumed two-player and multiplayer games. Phys Rev E 87, 062136 (2013).

35. Campenni, M. \& Schino, G. Partner choice promotes cooperation: The two faces of testing with agent-based models. J Theor Biol 344, 49-55 (2014).

36. Fu, F., Hauert, C., Nowak, M. A. \& Wang, L. Reputation-based partner choice promotes cooperation in social networks. Phys Rev E 78, 026117 (2008).

37. Wu, T., Fu, F. \& Wang, L. Partner selections in public goods games with constant group size. Phys Rev E 80, 026121 (2009).

38. Sylwester, K. \& Roberts, G. Cooperators benefit through reputation-based partner choice in economic games. Biol Lett 6, 659-662 (2010).

39. Li, Y., Min, Y., Zhu, X. \& Cao, J. Partner switching promotes cooperation among myopic agents on a geographical plane. Phys Rev E 87, 022823 (2013).

40. Li, Y. \& Shen, B. The coevolution of partner switching and strategy updating in non-excludable public goods game. Physica A 392, 4956-4965 (2013).

41. Hauert, C., Traulsen, A., Brandt, H., Nowak, M. A. \& Sigmund, K. Via freedom to coercion: the emergence of costly punishment. Science 316, 1905-1907 (2007).

42. Szabó, G. \& Töke, C. Evolutionary prisoner's dilemma on a square lattice. Phys Rev E 58, 69 (1998)

43. Currarini, S., Jackson, M. O. \& Pin, P. An Economic Model of Friendship: Homophily, Minorities, and Segregation. Econometrica 77, 1003-1045 (2009).

44. Ohtsuki, H., Hauert, C., Lieberman, E. \& Nowak, M. A. A simple rule for the evolution of cooperation on graphs and social networks. Nature 441, 502-505 (2006)
45. Shirado, H., Fu, F., Fowler, J. H. \& Christakis, N. A. Quality versus quantity of social ties in experimental cooperative networks. Nat Commun 4, 2814 (2013).

46. Eshel, I. \& Cavalli-Sforza, L. L. Assortment of encounters and evolution of cooperativeness. Proc Natl Acad Sci 79, 1331-1335 (1982).

47. Fu, F., Nowak, M. A., Christakis, N. A. \& Fowler, J. H. The evolution of homophily. Sci Rep 2, 845 (2012).

48. Coricelli, G., Fehr, D. \& Fellner, G. Partner selection in public goods experiments. I Confl Resolut 48, 356-378 (2004).

49. Page, T., Putterman, L. \& Unel, B. Voluntary association in public goods experiments: reciprocity, mimicry and efficiency. Econ J 115, 1032-1053 (2005).

50. Hauk, E. \& Nagel, R. Choice of partners in multiple two-person prisoner's dilemma games: an experimental study. J Confl Resolut 45, 770-793 (2001).

51. Barclay, P. \& Willer, R. Partner choice creates competitive altruism in humans. Proc R Soc B 274, 749-753 (2007).

52. Bashary, R. \& Grutter, A. S. Experimental evidence that partner choice is a driving force in the payoff distribution among cooperators or mutualists: the cleaner fish case. Ecol Lett 5, 130-136 (2002).

53. Christakis, N. A. \& Fowler, J. H. Friendship and natural selection. arXiv: 1308.5257 (2013)

54. de Nooy, W., Mrvar, A. \& Batagelj, V. Exploratory Social Network Analysis with Pajek: Revised and Expanded Second Edition (Cambridge University Press, 2011).

\section{Acknowledgments}

The author acknowledges the supports from the NSFC (11347201), the Zhejiang Provincial Natural Science Foundation (LQ13F030004) and the MOE (Ministry of Education in China) Project of Humanities and Social Sciences (13YJC630084).

\section{Additional information}

Competing financial interests: The authors declare no competing financial interests.

How to cite this article: $\mathrm{Li}, \mathrm{Y}$. The evolution of reputation-based partner-switching behaviors with a cost. Sci. Rep. 4, 5957; DOI:10.1038/srep05957 (2014).

This work is licensed under a Creative Commons Attribution-NonCommercialShareAlike 4.0 International License. The images or other third party material in this article are included in the article's Creative Commons license, unless indicated otherwise in the credit line; if the material is not included under the Creative Commons license, users will need to obtain permission from the license holder in order to reproduce the material. To view a copy of this license, visit http:// creativecommons.org/licenses/by-nc-sa/4.0/ 